\title{
The Promiscuous Pneumococcus---Evolution and Biology
}

\author{
Invited Lecture Abstracts
}

ISPPD-0533

The Promiscuous Pneumococcus---Evolution and Biology

\author{
HOW PNEUMOCOCCI COMPETE \\ S. Dawid ${ }^{1}$, T. Kochan ${ }^{2}$, W. Wholey ${ }^{1}$, N. LaCross ${ }^{1}$ \\ ${ }^{1}$ Pediatrics and Communicable Diseases, University of Michigan, Ann Arbor, USA; ${ }^{2}$ Microbiology and Immunology, University of Michigan, Ann Ar- \\ bor, USA
}

Competitive interactions between bacteria play an important role in shaping microbial communities on host surfaces. To survive antagonistic interactions in the nasopharynx, Streptococcus pneumoniae has developed offensive and defensive tactics that involve the production of lytic peptides called pneumocins and their associated immunity proteins. The production of pneumocins and immunity proteins is controlled by the extracellular accumulation of the signal peptide, BlpC. The locus encoding these proteins is characterized by significant allelic heterogeneity, in particular, in the genes encoding BlpC, pneumocin peptides and immunity proteins. This diversity reflects the impact of selective pressure from competing strains. Variability in immunity proteins provides a defensive strategy by allowing individual bacteria to avoid pneumocin mediated lysis. Because immunity tends to be restricted to specific pneumocins, defensive immunity can be offset by altering the repertoire of secreted peptides. Finally, production of distinct BIpC types restricts the broadcast of the "on" signal to only similarly responsive members of the population. This prevents the stimulation of neighboring strains that may be capable of producing a protective response. In addition to providing a direct competitive advantage during colonization, pneumocin secretion may also provide a means for genetic adaptation. Cross talk between the regulatory systems controlling pneumocin production and pneumococcal competence suggests that coordinate expression of the machinery required for horizontal gene transfer with the production of lytic peptides provides an adaptive advantage to pneumocin producing strains by increasing the pool of available genetic material.

No conflict of interest

Oral Plenary Abstracts

ISPPD-0374

The Promiscuous Pneumococcus---Evolution and Biology

THE IMPACT OF RECOMBINATION ON THE EMERGENCE OF NOVEL PNEUMOCOCCAL SEROTYPES

R. Mostowy ${ }^{1}$, N.J. Croucher ${ }^{1}$, C. Chewapreecha ${ }^{2}$, S. Salter ${ }^{2}$, P. Turner ${ }^{3}$, C. Turner ${ }^{3}$, L. Po $^{3}$, D. Goldblatt ${ }^{4}$, F. Nosten ${ }^{3}$, S. Bentley ${ }^{2}, X^{\prime}$ Didelot $^{1}$, C. Fraser ${ }^{1}$

${ }^{1}$ Department of Infectious Disease Epidemiology, Imperial College London, London, United Kingdom; ${ }^{2}$ Pathogen Genomics, Welcome Trust Sanger Institute, Hixton, United Kingdom; ${ }^{3}$ Tropical Medicine, Shoklo Malaria Research Unit, Maesot, Thailand; ${ }^{4}$ Immunobiology Unit, University College London, London, United Kingdom

Improving our knowledge of genetic transformation affecting the capsule biosynthesis locus is critical for better understanding of how pneumococci evolve in response to the introduction of vaccines targeting the bacteria's extracellular polysaccharides. Import of genetic polymorphisms at the polysaccharide locus has been observed in previous studies, but to what extent recombination can drive the evolution of serotypes remains not well understood. To study this process we examine the evolution within closely related, epidemiologically common serogroups 6, 19 and 23 from over 3,000 pneumococcal isolates collected in a refugee camp in Mae La, Thailand. To identify a clonal phylogeny for each of those serogroups we identify dense clusters of polymorphisms on each branch of a phylogenetic tree and assume them to be recombinations. By removing them, we arrive at clonal phylogenies of the studied serogroups, with recombination and mutation events mapped on each tree. This picture shows a strong impact of recombination on the emergence of capsular diversity, particularly in serogroup 6. Interestingly, it suggests that some serotypes have emerged multiple times, ie against independent clonal backgrounds, a scenario likely driven by the selective pressure of the immune system. Comparison of the inferred recombination sequences with the sequences of all 92 serotype sequences suggests other serogroups and nonpneumococcal species as likely sources of genetic diversity. Overall, this study suggests that the adaptive potential of recombination in Streptococcus pneumoniae may be even greater than currently assumed.

No conflict of interest 


\section{GENOME SEQUENCING OF A GLOBAL COLLECTION OF SEROTYPE 1 PNEUMOCOCCI, REVEALS SEROTYPE 1 IS} GENETICALLY DISTINCT BETWEEN COUNTRIES

J. Cornick ${ }^{1}$, S. Harris ${ }^{2}$, Y. Feyruz ${ }^{2}$, C. Chaguza ${ }^{1}$, M. Senghore ${ }^{3}$, M. Antonio ${ }^{3}$, J.M. Collard ${ }^{4}$, N. French ${ }^{5}$, R. Heyderman ${ }^{1}$, K. Klugman', A. Von Gottberg ${ }^{7}$, S. Bentley ${ }^{2}$, D. Everett ${ }^{1}$

${ }^{1}$ Microbial Genomics, Malawi-Liverpool-Wellcome Trust Clinical Research Programme, Blantyre, Malawi; ${ }^{2}$ Pathogen Genomics, Wellcome Trust Sanger Institute, Hinxton, United Kingdom; ${ }^{3}$ Bacterial Disease Programme, Medical Research Council Unit the Gambia, Fajara, Gambia ${ }^{4}$ Laboratoire de Bacteriologie, Centre de Recherche Médicale et Sanitaire, Niamey, Niger; ${ }^{5}$ Institute of Infection and Global Health, University of Liverpool, Liverpool, United Kingdom; ${ }^{6}$ Hubert Department of Global Health, Emory University, Atlanta, USA; ${ }^{7}$ National Institute of Infectious Diseases, National Institute of Health Research, Johannesburg, South Africa

Background and Aims: In Africa, serotype 1 is the second most common cause of invasive pneumococcal disease after serotype 14. Serotype 1 is a unique serotype; it is rarely isolated in carriage but has a high likelihood to cause invasive disease and is associated with epidemic outbreaks in West Africa. We aimed to define the genetic diversity of serotype 1 worldwide.

Methods: 448 serotype 1 isolates, from 28 countries (11 African), were sequenced on the Illumina GA11/Ilumina HiSeq2000. Sequence reads were mapped to a Ghanaian reference using SMALT, variant sites in the core genome identified and RAxML was used to construct a phylogeny.

Results: The phylogenetic reconstruction of serotype 1 showed strong continental clustering. Within the African lineage there was strong country specific structuring with strains from each collection site forming distinct clades separated by a large number of single-nucleotide polymorphisms (SNPs).

Isolates from carriage and invasive disease groups did not form separate clusters on the phylogeny, suggesting a similar genetic background in carriage and disease. Similarly isolates from adults and children did not cluster separately, suggesting the same genotype is circulating between adults and children.

Conclusion: Serotype 1 isolates in Africa are genetically distinct from elsewhere in the world. The short duration of carriage, arguably limits cross continent transfer. This has potentially important implications when designing control strategies. Furthermore, within Africa there are distinct genetic differences between West and East. By comparing the core genome of isolates from the two regions, we may identify genes specifically associated with meningitis outbreaks.

Conflict of interest

ISPPD-0103

The Promiscuous Pneumococcus---Evolution and Biology

\section{CYTOSOLIC ACCESS OF PNEUMOCOCCAL CELL WALL COMPONENTS IN MACROPHAGES IS DEPENDENT ON PNEUMOLYSIN}

J. Lemon ${ }^{1}$, J. Weiser ${ }^{1}$

${ }^{1}$ Microbiology, University of Pennsylvania Perelman School of Medicine, Philadelphia, USA

Background and Aim: The extracellular pathogen Streptococcus pneumoniae serially inhabits the human nasopharynx in a dynamic process of colonization, resulting in innate immune signaling and subsequent bacterial clearance. Intracellular detection of pneumococcal cell wall fragments by the host cytosolic receptor NOD2 is required for effective clearance of colonization and is dependent on phagocytosis, and the pneumococcal poreforming toxin pneumolysin. We hypothesize that after uptake, killing, and degradation in the phagolysosome, pneumococcal cell wall components escape into the host-cell cytosol through pneumolysin pores in the phagosome membrane.

Methods: We monitored access to the host-cell cytosol by following the transit of pneumococcal capsular polysaccharide. Confocal microscopy, immuno-fluorescence staining, and electron microscopy were used to visually characterize the location of pneumococcal cell wall components in murine macrophages following phagocytosis. The amount of bacterial cell wall components in the cytosol was quantified through detergent-based cellular fractionation, followed by capture ELISA.

Results: We observed foci of bacterial components, which do not co-localize with lysosomes. Electron microscopy showed that these cell wall components are within the cytosol, outside of membrane-bound organelles. Quantification by ELISA found that $>10 \%$ of pneumococcal components are present in the cytosol within 2 hours and this access is dependent on bacterial uptake. Cell wall components from a pneumococcal strain deficient in pneumolysin had significantly reduced access to the host-cell cytosol.

Conclusion: Our results show that access of pneumococcal components to the host-cell cytosol is dependent on phagocytosis and pneumolysin. This suggests its pore-forming toxin mediates immune detection akin to an intracellular pathogen.

No conflict of interest 
ISPPD-0557

The Promiscuous Pneumococcus---Evolution and Biology

\title{
CAPSULAR POLYSACCHARIDE RELEASE BY TYPE 3 PNEUMOCOCCAL STRAINS MAY EXPLAIN THE LACK OF PROTECTION BY CONJUGATE VACCINE
}

\author{
E. Choi ${ }^{1}$, F. Zhang ${ }^{1}$, Y.J. Lu ${ }^{1}$, R.M. Malley ${ }^{1}$ \\ ${ }^{1}$ Division of Infectious Diseases, Children's Hosp/Harvard Medical School, Boston, USA
}

Background and Aims: Concern has been raised about the efficacy of the serotype 3 pneumococcal conjugate vaccine (PCV). The type 3 and 37 capsular polysaccharides (CPS) are synthesized through a synthase-dependent pathway, unlike all other serotypes that use the wzy-dependent pathway. This could result in a significant release of serotype (ST) 3 CPS during growth which we hypothesized may reduce vaccine efficacy.

Methods: CPS release was measured by inhibition ELISA. Opsonophagocytic assays (OPAs) were performed with differentiated HL-60 cells. Mice were injected with anticapsular antibodies and challenged intraperitoneally (IP) with or without culture supernatant.

Results: In vitro, mean released CPS concentrations per $10^{7} \mathrm{CFU}$ of ST $3(60 \mu \mathrm{g} / \mathrm{mL})$ and $37(130 \mu \mathrm{g} / \mathrm{mL})$ strains were significantly higher than ST 1, 4, 6B, and $14(0.4-10 \mu \mathrm{g} / \mathrm{mL})$. Following IP injection, concentrations of released CPS in blood were $31.2 \mu \mathrm{g} / \mathrm{mL}$ for ST3, $<0.1 \mu \mathrm{g} / \mathrm{mL}$ for ST4 $(p=0.0159)$, and $0.8 \mu \mathrm{g} / \mathrm{mL}$ for ST5 $(p<0.008$ for comparisons of ST3 with others). Opsonophagocytic killing of anti-ST3 antibody was significantly reduced by addition of ST3 CPS, culture supernatant or serum from ST3-infected mice. Protection by passive transfer of antibody was significantly inhibited when $0.2 \mu$ l of culture supernatant was added in the IP challenge model.

Conclusion: Released ST3 CPS interferes with antibody-mediated killing of ST3 pneumococci and protective effects of anti-CPS antibody in vivo. The failure of PCVs in protection against ST3 may be due to the CPS release rather than low immunogenicity, suggesting that alternative approaches for the control of ST3 may be necessary.

No conflict of interest

\section{Oral Poster Abstracts}

ISPPD-0140

The Promiscuous Pneumococcus---Evolution and Biology

\section{DISCOVERY OF STREPTOCOCCUS PNEUMONIAE SEROGROUP 6 VARIANTS WITH BI-SPECIFIC WCINALPHA}

\author{
M.B. Oliver ${ }^{1}$, M. van der Linden ${ }^{2}$, S.A. Küntzel ${ }^{2}$, J. Saad $^{3}$, M.H. Nahm ${ }^{4}$ \\ ${ }^{1}$ Department of Microbiology, University. of Alabama at Birmingham, Birmingham AL, USA; ${ }^{2}$ National Reference Center for Streptococci Depart- \\ ment of Medical Microbiology, University Hospital RWTH Aachen, Aachen, Germany; ${ }^{3}$ Department of Microbiology, University of Alabama at Bir- \\ mingham, Birmingham AL, USA; ${ }^{4}$ Department of Microbiology Department of Pathology, University of Alabama at Birmingham, Birmingham AL, \\ USA
}

Background and Aims: Genetic variation of the capsular-polysaccharide-synthesis (cps) locus is the molecular basis for structural and antigenic heterogeneity of the 94 known serotypes of Streptococcus pneumoniae. Serogroup 6 has 4 members with distinct serological properties, homologous cps loci and structurally similar polysaccharides (PS). 6A and 6B have wciN $\alpha$, encoding $\alpha$-1,3-galactosyl-transferase, $6 \mathrm{C}$ and $6 \mathrm{D}$ have wciN $\beta$ encoding $\alpha$-1,3-glucosyltransferase. Recently, two serogroup 6 clinical isolates with wciN $\alpha$ but serologic properties of 6C and 6D recovered in Germany were discovered using the Quellung method.

Methods and Results: The strains $(6 \times 12,6 \times 11)$ were confirmed to have unique serologic properties of $6 \mathrm{~A} / 6 \mathrm{C}$, and also 6B/6D using serogroup 6-specific mouse mAbs. Capsular-PS was purified and analyzed by NMR revealing that 6X12 PS had two different repeating units: 75\% 6A-PS and 25\% 6C-PS. 6X11-PS also contained two different repeating units: $40 \% 6 \mathrm{~B}$ and $60 \% 6 \mathrm{D}$. Genetic studies revealed mutations in wciNa. $6 \mathrm{X} 12$ had one mutation (A150T) and $6 \times 11$ had two mutations (D38N,A150T). When A150T mutation was introduced to the cps of a 6A strain by sitedirected mutagenesis, the mutant (MBO172) had identical serological profiles to 6X12. The structure of MBO172 PS is currently being investigated by nuclear magnetic resonance (NMR) spectroscopy.

Conclusion: Single amino acid changes in cps genes encoding glycosyl-transferases can alter substrate specificities, permit biosynthesis of heterogeneous capsule repeating units, and result in new capsule types within a welldefined serogroup. Thus, immunological pressure from widespread pneumococcal vaccination may select variants expressing novel non-vaccine capsule types and multiple analytical approaches may be required for accurate identification of capsule types.

No conflict of interest 
ISPPD-0356

The Promiscuous Pneumococcus---Evolution and Biology

\section{GENETIC RECOMBINATION AND NASOPHARYNGEAL CARRIAGE DYNAMICS IN PNEUMOCOCCAL SEROTYPE-1 STRAINS FROM AFRICA}

C. Chaguza ${ }^{1}$, J.E. Cornick ${ }^{1}$, C. Chewapreecha ${ }^{2}$, F. Yalcin ${ }^{2}$, M. Senghore 3 , S. Govindpersad ${ }^{4}$, A. Von Gottberg ${ }^{4}$, J. Collard $^{5}$, K.P. Klugman', M. Antonio ${ }^{3}$, N. French', R.S. Heyderman' ${ }^{1}$, S.R. Harris², S.D. Bentley², D.B. Everett ${ }^{1}$

${ }^{1}$ Microbes Immunity and Vaccines, Malawi-Liverpool-Wellcome Trust Clinical Research Programme, Blantyre, Malawi; ${ }^{2}$ Pathogen Genomics, Wellcome Trust Sanger Institute, Cambridge, United Kingdom; ${ }^{3}$ Bacterial Disease Programme, Medical Research Council Unit, Fajara, Gambia; ${ }^{4}$ Respiratory and Meningeal Pathogens Research Unit, National Health Laboratory Services, Johannesburg, South Africa; ${ }^{5}$ Laboratoire de Bacteriologie, CERMES Reseau International des Instituts Pasteur, Niamey, Niger; ${ }^{6}$ Hubert Department of Global Health, Rollins School of Public Health, Emory University, Georgia, USA; ${ }^{7}$ Institute of Infection and Global Health, University of Liverpool, Liverpool, United Kingdom

Background and Aims: Streptococcus pneumoniae serotype 1 ranks among the most prevalent invasive pneumococcal serotypes worldwide, particularly in the developing countries. Although carriage is seen as a prerequisite for both invasive disease and genetic recombination, serotype 1 is rarely detected in the nasopharyngeal carriage. The rarity in detection during colonisation drove the assumption that it rarely undergoes recombination since it occurs exclusively in carriage. We aimed to test the hypothesis that recombination does not occur in serotype 1 strains and to establish its association to the geographical origin of the strains and its consequence to nasopharyngeal carriage.

Methods: We analysed 448 Illumina sequenced genomic-DNA libraries collected through the Pneumococcal African Genomics (PAGe) Consortium using a novel approach for detecting recombinant DNA fragments by statistical evaluation of polymorphism density in aligned sequences.

Results: Almost 23965 segregating (polymorphic) sites were identified and $\geq 80 \%$ of these emerging through $>300$ genetic recombination exchanges. Genes encoding antimicrobial resistance targets were also frequently involved in recombination. Furthermore, we observed a high geographical clustering, clonal population structure and variation in the levels of recombination between geographically distinct strains.

Conclusion: The presence of both ancestral and recent genetic recombination events suggests its role in the evolution of serotype 1 strains. The findings further suggest that the rarity in the nasopharyngeal carriage could explain the observed slow acquisitions of DNA as a consequence of limited exposures. Variation in the levels of recombination is driven by the differences in selective pressures in different locales.

No conflict of interest

ISPPD-0282

The Promiscuous Pneumococcus---Evolution and Biology

\section{EXPERIMENTAL HUMAN PNEUMOCOCCAL CARRIAGE IS DEPENDENT ON THE PEPTIDE PERMEASE amiC}

J.F. Gritzfeld ${ }^{1}$, A.B. Dalia ${ }^{2}$, D.M. Ferreira ${ }^{1}$, A.M. Roche ${ }^{3}$, S. Glennie ${ }^{4}$, S.H. Pennington ${ }^{1}$, M. Bangert ${ }^{5}$, J.E. Cornick $^{6}$, A.D. Wright ${ }^{1}$, A.M. Collins ${ }^{1}$, A. Camilli ${ }^{2}$, J.N. Weiser ${ }^{3}$, D.B. Everett ${ }^{6}$, A. Kadioglu ${ }^{7}$, S.B. Gordon ${ }^{1}$

\footnotetext{
${ }^{1}$ Respiratory Infection Group, Liverpool School of Tropical Medicine, Liverpool, United Kingdom; ${ }^{2}$ Howard Hughes Medical Institute and Department of Molecular Biology and Microbiology, Tufts University School of Medicine, Boston, USA; ${ }^{3}$ Department of Microbiology, University of Pennsylvania School of Medicine, Philadelphia, USA; ${ }^{4}$ Cellular and Molecular Medicine School of Medical Sciences, University of Bristol, Bristol, United Kingdom; ${ }^{5}$ Respiratory Infection, Liverpool School of Tropical Medicine, Liverpool, United Kingdom; ${ }^{6}$ Malawi-Liverpool-Wellcome Clinical Research Programme, University of Malawi College of Medicine, Blantyre, Malawi; ${ }^{7}$ Department of Clinical Infection Microbiology and Immunology Institute of Infection and Global Health, University of Liverpool, Liverpool, United Kingdom
}

Background and Aims: The differing propensity of pneumococcal strains to establish carriage is of importance in transmission and disease. We compared two strains (6B and 23F) with previously similar carriage rates in human challenge experiments and found reduced carriage of the $23 \mathrm{~F}$ strain.

Methods: 60 healthy adults were intranasally inoculated with type $6 \mathrm{~B}$ and 60 adults with type 23F Streptococcus pneumoniae in dose ranging studies. Carriage rates detected by nasal wash and microbiological culture were compared. The strains were also compared in vitro for colony morphology, adherence to cultured nasopharyngeal epithelial cells, complement deposition and opsonophagocytic killing. The whole genome sequences of both strains were also compared.

Results: There was markedly greater colonisation of human volunteers by type 6B compared to $23 \mathrm{~F}(\mathrm{p}<0.0001)$. The $6 \mathrm{~B}$ strain showed greater adherence than $23 \mathrm{~F}$ using nasopharyngeal epithelial cells $(p=0.03)$ and was less susceptible to complement deposition $(p<0.001)$. There was no difference in opsonophagocytic killing ( $p=0.1)$. Two genes, $p c p \mathrm{~A}$ and aliB, were unique to $6 \mathrm{~B}$ while the $23 \mathrm{~F}$ strain had an amiC frameshift mutation. The amiC mutation was demonstrated to drastically reduce carriage in a mouse model.

Conclusion: Dramatic differences in carriage rates were associated with differences in protein expression particularly amiC, $p c p A$ and aliB. Murine models and previous human studies suggest that amiC is the most significant of these factors.

No conflict of interest 


\title{
EXPRESSION OF CHOLINE BINDING PROTEINS IN STREPTOCOCCUS PNEUMONIAE STRAINS INDUCED WITH PENICILLIN STRESS
}

\author{
N.K. Palanisamy ${ }^{1}$, E.H. Wong ${ }^{2}$, P. Navaratnam ${ }^{3}$, S.D. Sekaran ${ }^{4}$ \\ ${ }^{1}$ Microbiology, Universiti Teknologi MARA, Sungai Buloh, Malaysia; ${ }^{2}$ School of Medicine, Taylor's University Lakeside Campus, Subang Jaya, Ma- \\ laysia; ${ }^{3}$ School of Medicine and Health Sciences, Monash University, Sunway, Malaysia; ${ }^{4}$ Medical Microbiology, University of Malaya, Kuala Lum- \\ pur, Malaysia
}

Background: Invasive pneumococcal disease is a major cause of morbidity and mortality, presenting invasive infections such as lobar pneumonia, bacteremia and meningitis. The increasing incidence of penicillin resistance in Stretococcus pneumoniae is a growing concern. The evolution of this species of bacteria has enabled it to develop resistance to many other antibiotics.

Aim: In this study, the Affymetrix Genechip microarray was employed to investigate the role of a wide range of genes involved in the development of penicillin resistance.

Methods: Strains with different susceptibilities to penicillin was extracted before and after been exposed to penicillin stress. The respective cDNA was hybridized onto gene chip expression arrays masked with probes representing the known genome of S. pneumoniae TIGR4.

Results: Genes encoding choline binding proteins (CBPs) showed significant levels of expression. $c b p$ gene showed an increased mRNA expression level in the penicillin resistant strain with a 4.96 fold, whereas the $c b p$ genes $A, D$, $J, G$ and $F$ showed decreased expression levels in the penicillin resistant strain upon penicillin stress. No significant change was observed in the expression levels of $\operatorname{cbps} A, C, J, G$ and $F$ in the penicillin sensitive strain. This suggests that the decreased expression of these genes in the resistant strain reduces its capacity to undergo cell separation and autolysis, hence failing to lyse and become tolerant.

Conclusion: Resistance to penicillin might be due to impaired autolytic pathway which may have been modified with various adaptive regulatory mechanisms within the bacterial cell.

No conflict of interest

ISPPD-0450

The Promiscuous Pneumococcus---Evolution and Biology

\section{STRUCTURAL AND FUNCTIONAL CHARACTERIZATION OF A PNEUMOCOCCAL SURFACE PROTEIN THAT BINDS TO HUMAN FACTOR H}

\author{
A. Pathak ${ }^{1}$, T. Schulte ${ }^{2}$, A. Achour ${ }^{2}$, S. Browall ${ }^{1}$, S. Normark' ${ }^{1}$, B.H. Normark ${ }^{1}$ \\ ${ }^{1}$ Microbiology and tumor cell biology, Karolinska Institute, Stockholm, Sweden; ${ }^{2}$ Center for Infectious Medicine, Karolinska Institute, Stockholm, \\ Sweden
}

Streptococcus pneumoniae can invade host immune system with the help of virulence factors that interact directly with host cellular receptors. One such virulence factor is Pneumococcal surface protein C (PspC). Whole genome sequence analyses of various clinical isolates revealed the presence of two different $p s p$ Cenes in few strains, $p s p C 1$ encoding a choline binding version and $p s p C 2$ encoding a cell wall anchored LPXTG protein. Interestingly, PspC2 displayed an overall low sequence homology to various PspC proteins which are known to interact with human Factor $\mathrm{H}(\mathrm{FH})$. Further investigations showed that despite of lacking the conserved hexapeptide motif known to bind FH, PspC2 binds to human FH through its amino terminal domain. Unlike other members of the PspC protein family, PspC2 utilizes a novel sequence of amino acids for this interaction. Here we defined a novel FH binding motif utilized by $\mathrm{PspC} 2$ to interact with human $\mathrm{FH}$. Further structural and biochemical investigations will provide more insights into the molecular basis of PspC2-FH interaction and its role in pneumococcal disease.

No conflict of interest

\section{ISPPD-0156}

The Promiscuous Pneumococcus---Evolution and Biology

\section{THIOL PEROXIDASE MEDIATES STREPTOCOCCUS PNEUMONIAE RESPONSES TO OXIDATIVE STRESS}

\author{
B. Hajaj ${ }^{1}$, H. Yesilkaya ${ }^{2}$, S. Shafeeq ${ }^{3}$, R. Benisty ${ }^{1}$, P.W. Andrew ${ }^{2}$, O.P. Kuipers ${ }^{4}$, N. Porat ${ }^{1}$
}

${ }^{1}$ Pediatric Infectious Disease Unit Department of Microbiology and Immunology, Soroka University Medical Center and Ben-Gurion University of the Negev, Beer-Sheva, Israel; ${ }^{2}$ Infection Immunity \& Inflammation, University of Leicester, Leicester, United Kingdom; ${ }^{3}$ Department of Microbiology Tumor and Cell Biology, Karolinska Institute, Stockholm, Sweden; ${ }^{4}$ Department of Molecular Genetics, University of Groningen, Groningen, Netherlands

Background and Aims: The psaBCAD operon of Streptococcus pneumoniae encodes an ABC-Mn ${ }^{2+}$-permease complex ( $s a \mathrm{BCA}$ ) and thiol peroxidase (tpxD), which may be co-transcribed. We have recently shown that TpxD expression is modulated in accordance with $\mathrm{H}_{2} \mathrm{O}_{2}$ concentrations, and that TpxD plays a role in the precise control of $\mathrm{H}_{2} \mathrm{O}$ levels. This study aimed to show that TpxD mediates pneumococal responses to $\mathrm{H}_{2} \mathrm{O}_{2}$.

Methods: Wild-type D39, D39 $\Delta$ tpxD and D39 $\Delta$ psaR were used. TpxD expression was determined by real-time PCR (RT-PCR) and immunoblotting. Global gene expression was checked by microarray analysis. Detection of reversibly oxidized bacterial cysteine residues was done by using biotinylated iodoacetamide followed by immunoblotting. 
Results: Challenging D39 with $\mathrm{H}_{2} \mathrm{O}_{2}$ resulted in psaBCA down-regulation while tpxD expression was up-regulated. However, in $\Delta t p x D$ and $\Delta p s a \mathrm{R}$-mutants, $\mathrm{H}_{2} \mathrm{O}_{2}$ had no effect on psaBCA expression, implying that both TpxD and PsaR are involved in the effect of $\mathrm{H}_{2} \mathrm{O}_{2}$ on the psa operon. Microarray analysis revealed that in D39 challenged with $\mathrm{H}_{2} \mathrm{O}_{2}, 148$ genes were differentially expressed, of which 16 were transcriptional regulators. Conversely, $\mathrm{H}_{2} \mathrm{O}_{2}$ had only marginal effect on the transcriptome of D39 $\Delta$ tpxD-mutant, indicating that TpxD mediates the pneumococcal response to $\mathrm{H}_{2} \mathrm{O}_{2}$. Furthermore, the level of reversibly oxidized cysteine residues in protein extracts of $\mathrm{H}_{2} \mathrm{O}_{2}-$ challenged bacteria was higher compared to untreated bacteria, while in the $\Delta t p x \mathrm{D}$-mutant there was almost no effect.

Conclusions: Our data suggest that TpxD exhibits two functions associated with $\mathrm{H}_{2} \mathrm{O}_{2}$ reduction: (i) It transmits oxidative signals to upstream effectors, such as transcription factors; (ii) It provides antioxidant defense by direct reduction of $\mathrm{H}_{2} \mathrm{O}_{2}$.

No conflict of interest

ISPPD-0123

The Promiscuous Pneumococcus---Evolution and Biology

\title{
QUANTIFICATION OF TRANSCRIPTS OF STREPTOCOCCUS PNEUMONIAE VIRULENCE GENES AND VACCINE CANDIDATES IN THE NASOPHARYNX OF HEALTHY CHILDREN
}

\author{
F. Sakai ${ }^{1}$, S.J. Talekar ${ }^{1}$, C.F. Lanata ${ }^{2}$, C.G. Grijalva ${ }^{3}$, Griffin M.R. ${ }^{3}$, Edwards K.M. ${ }^{4}$, K.P. Klugman ${ }^{1}$, J.E. Vidal ${ }^{1}$ \\ ${ }^{1}$ Hubert Department of Global Health, Emory University, Atlanta, USA; ${ }^{2}$ Infectious Diseases, Instituto de Investigacion Nutricional, Lima, Peru; \\ ${ }^{3}$ Department of Preventive Medicine, Vanderbilt University School of Medicine, Nashville, USA; ${ }^{4}$ Vanderbilt Vaccine Research Program, Vander- \\ bilt University School of Medicine, Nashville, USA
}

Background: Colonization and persistence in the human nasopharynx are prerequisites for Streptococcus pneumoniae (Sp) disease and carriage, which normally occurs during early childhood. Studies related to the mechanism of persistence have revealed a number of colonization and virulence factors as well as regulators implicated in nasopharyngeal colonization and pathogenesis. Expression of genes encoding these factors has never been studied in the human nasopharynx. Therefore, this study developed the technology to detect $S p$ genes in human nasopharyngeal samples and quantified mRNA transcripts of genes shown to be important for persistence and virulence.

Methods: Nasopharyngeal samples were collected from healthy children $<3$ years of age in Peru. DNA and total RNA were extracted with commercial kits (Qiagen). A panel of 21 primers that amplified Sp sequences were designed, in silico analyzed, and their specificity was validated against 20 related strains present in the human nasopharynx. Results: Primers were utilized in conventional PCR, quantitative (q)PCR reactions to find that $100 \%$ of samples contained the genes ply, lytC, pavA, lytA, comD, codY and mgrA, whereas the following set of genes were present in $>90 \%$ of samples including nanA, nanB, pspA, and $r r g$ B. Gene expression studies, (q)RT-PCR, of these 11 targets revealed that lytC, lytA and nanB are highly expressed whereas all others, except rrgB, have a moderate to low level of expression.

Conclusion: This is the first study to evaluate expression of virulence, colonization and regulator related genes in the nasopharynx of healthy children and highlights the importance of lytC in nasopharyngeal persistence.

No conflict of interest

ISPPD-0159

The Promiscuous Pneumococcus---Evolution and Biology

\section{POPULATION DYNAMICS OF STREPTOCOCCUS PNEUMONIAE VACCINE SEROTYPES ON HUMAN PHARYNGEAL CELLS}

J.E. Vidal ${ }^{1}$, F. Sakai ${ }^{1}$, J. Concepción-Acevedo ${ }^{2}$, J.R. Shak ${ }^{1}$, S. Chochua ${ }^{1}$, K.P. Klugman ${ }^{1}$

${ }^{1}$ Hubert Department of Global Health, Emory University, Atlanta, USA; ${ }^{2}$ Department of Biology, Emory University, Atlanta, USA

Background: Streptococcus pneumoniae (Sp) persists in the human nasopharynx forming biofilms. Nearly half children carry at least 2 different serotypes. On abiotic surfaces, biofilms are regulated by the quorum sensing system LuxS/AI-2. However, virtually nothing is known about biofilms grown on human cells.

Methods: Our well established biofilm bioreactor (biBio), with human pharyngeal cells, was utilized to simulate the microenvironment $S p$ finds in the respiratory epithelium. biBio was infected with a mixture of two vaccine types (2SsBs). The structure and population dynamics of $2 \mathrm{SsBs}$ were studied.

Results: Biofilms formed on human cells maintained their population, average of $\sim 5.6 \times 10^{8} \mathrm{CFU}$ of biofilm cells/ $\mathrm{cm}^{2}, 24 \mathrm{~h}$ post-inoculation. Planktonic/invasive $S p$ cells coming off the biofilm structure remained stable $\left(\sim 1.98 \times 10^{6}\right.$ CFU/ml). Populations of D39 $/$ luxS, D39 $\Delta$ comC or D39 $\Delta$ ply were significantly lower than wt biofilms. Transcriptional upregulation of important genes was observed in biofilms grown on human cells (i.e. lytA, psaA, pspA and ply). Ply was located on the cell wall and the biofilm matrix. Mixtures of two vaccine serotypes (similar or different CSPpherotype, e.g. 4, 6B, 19A, 19F or 23F) were inoculated into the biBio to find out three population dynamics: 1) biomass of both vaccine types remained similar, 2) population of a serotype predominated (fitness advantage) or 3 ) persistence of a serotype eradicated the other strain. Confocal microscopy studies revealed fused 2SsBs structures made by a mixture of specific serotypes.

Conclusion: Population dynamics of $S p$ vaccine types is fine-tuned by specific mechanisms allowing, or not, persistence of $S p$ strains on human pharyngeal cells. 


\title{
Poster Abstracts
}

ISPPD-0219

The Promiscuous Pneumococcus---Evolution and Biology

\section{STREPTOCOCCUS PNEUMONIAE SEROTYPE 1'S ESCAPE FROM DETECTION IN CARRIAGE: A GENETIC MECHANISM?}

\author{
M. Alaerts ${ }^{1}$, E. Heinsbroek ${ }^{2}$, A. Chirambo ${ }^{1}$, A. Phiri ${ }^{3}$, N. French ${ }^{2}$, D.B. Everett ${ }^{1}$ \\ ${ }^{1}$ Microbial Genomics, Malawi-Liverpool-Wellcome Trust Clinical Research Programme, Blantyre, Malawi; ${ }^{2}$ Department of Clinical Infection Mi- \\ crobiology and Immunology, Institute of Infection and Global Health, Liverpool, United Kingdom; ${ }^{3}$ Laboratory Department, Karonga Preven- \\ tion Study, Chilumba, Malawi
}

Background: Streptococcus pneumoniae Serotype 1 causes a high proportion of invasive pneumococcal disease (IPD), particularly in developing countries, yet it is rarely detected in healthy carriers. This may occur because it is carried for very short periods of time and transmission rates are high or alternatively, because it goes undetected in carriage. We hypothesized that Serotype 1 has the capacity to switch between a non-encapsulated carriage state colonizing the nasopharynx and an encapsulated virulent state causing IPD, through genetic recombination. Deletion and re-insertion of the functional portion of the Serotype $1 \mathrm{cps}$ locus through homologous recombination involving the flanking IS1167 sequences could provide such a switch mechanism. This deletion was recently detected in 4 nontypeable isolates from Native American communities.

Methods: Monthly nasopharyngeal swabs were taken from each member of households participating in a longitudinal carriage study in Karonga, Malawi. Households with at least one Serotype 1 carriage event were recruited to this study. Molecular methods were used to test for the presence of the Serotype 1 deletion variant in all pneumococci positive swabs.

Results: Five households were selected with a total of 112 swabs available for testing. Pneumococcal growth was obtained from 65 swabs. Six swabs were Serotype 1 positive. The deletion variant was not detected.

Conclusion: We did not detect the Serotype $1 \mathrm{cps}$ deletion variant in this pilot study. We plan a larger study which includes nasopharyngeal aspirates and will use DNA extracted directly from samples without a culturing step to increase sensitivity.

No conflict of interest

ISPPD-0055

The Promiscuous Pneumococcus---Evolution and Biology

\section{PREVALENCE OF VARIOUS SEROTYPES OF STREPTOCOCCUS PNEUMONIAE ISOLATED FROM CLINICAL SAMPLES}

L. Anantha Raman ${ }^{1}$, V.L. Malhotra ${ }^{1}$

${ }^{1}$ Microbiology, Lady Hardinge Medical College and Associated Hospitals, Delhi, India

Background and Aims: The aim of the present study was to find out the prevalence of various serogroups/types of Streptococcus pneumoniae (SP) isolated from clinical samples.

Methods: A total of 544 strains of $S$. pneumoniae isolated from patients aged 1 mo-60 yr attending the outpatient Department of KSCH and Smt .S.K. Hospital, New Delhi, India during 1988-1997 were biochemically characterized using standard procedures. The serotyping of the strains was carried out using Quellung reaction using type specific pneumococcal antisera.

Results: S. pneumoniae strains (86.4\%) were found to be typable and they constituted 18 serogroups/types. The percentage of SP strains isolated in children ( $<13 \mathrm{yr})$ and adults/adolescents ( $>13 \mathrm{yr})$ from CSF and blood were 31.3 and 13.2 and 12.1 and 20.2 respectively. The other samples that yielded SP were pus (17.5\%), Sputum/ bronchial aspirate/gastric aspirate (17.1\%), peritoneal fluid (3.5\%), conjunctival swab (2.4\%), and ear swab (1.8\%). The most common serogroups/types encountered in the present study were type $1(32.3 \%), 3(16.5 \%), 19(10.3 \%)$ followed by $5(8.0 \%), 15(7.6 \%), 6(6.8 \%), 7(6.5 \%), 27(5.1 \%), 13(4.6 \%), 23(3.8 \%), 9(2.3 \%), 14(2.0 \%), 18,12,29,37$ and $39(1.1 \%)$ each and $21(0.6 \%) .10$ out of 18 serogroups/types isolated in the present study are included in the $23-$ valent pneumococcal vaccine. All the strains were found to be sensitive to Penicillin.

Conclusion: The study emphasizes the need for continued epidemiological surveillance of pneumococcal infections as it helps to monitor regularly the prevalent serogroups/types of $S$. pneumoniae circulating in the community. In the future for the development of a safe successful polyvalent vaccine suitable for Indian conditions should include locally prevalent serogroups/types known to cause invasive infections in children and adults.

No conflict of interest 
ISPPD-0054

The Promiscuous Pneumococcus---Evolution and Biology

\section{DISTRIBUTION OF STREPTOCOCCUS PNEUMONIAE SEROTYPES CAUSING EYE INFECTIONS}

L. Anantha Raman ${ }^{1}$, V.L. Malhotra ${ }^{1}$

${ }^{1}$ Microbiology, Lady Hardinge Medical College and Associated Hospitals, Delhi, India

Background and Aims: The aim of the present study was to find out the distribution of Streptococcus pneumoniae serotypes causing eye infections. The antimicrobial susceptibility pattern of the strains was carried out to find out the prevalence of drug resistance among the commonly used antibiotics.

Methods: A total of 176 strains of $S$. pneumoniae isolated from patients aged $1 \mathrm{~m}-60 \mathrm{yr}$ attending the outpatient Department of Eye of KSCH and Smt .S.K. Hospital, New Delhi, India during 1981-1995 were biochemically characterized using standard procedures. The serotyping of the strains was carried out using Quellung reaction using type specific pneumococcal antisera. The antibiotic susceptibility pattern of these strains was determined by disk diffusion method.

Results: S. pneumoniae strains (96.6\%) were found to be typable and they constituted 16 serogroups/types. The most common serotype was $3(19.6 \%)$ followed by $12(15.3 \%), 6(13.6 \%), 33(11.4 \%), 28(8.0 \%), 4.5 \%$ each of 19 and $46,45(3.4 \%)$ and $2.8 \%$ each of $11,13,23,40(2.3 \%), 18,29(, 1.7 \%), 1.1 \%$ each of 8 and 21 . The eye infections caused by these strains were dachryocystitis ( $47.2 \%)$, conjunctivitis (45.5\%), endopthalmitis $(4.0 \%)$ and lacrymal gland infection (3.4\%). All the strains were sensitive to penicillin, ampicillin, cloxacillin chloramphenicol, erythromycin, co-trimoxazole, gentamicin and streptomycin. There were 2 strains, 1 each of serotype 3 (endopthalmitis) and 12 (conjunctivitis) resistant to tetracycline.

Conclusion: The study highlights the importance of serotypes causing eye infections as information regarding their distribution is scanty. There were no penicillin resistant strains causing eye infections during 1981-1995. But there were 2 tetracycline resistant strains 1, type 3 causing endopthalmitis and type 12 causing conjunctivitis. Comparative studies with other areas of the world may help in further understanding our observations, as bacterial virulence factors and host factors may play an important role in the selection of serotypes

No conflict of interest

ISPPD-0233

The Promiscuous Pneumococcus---Evolution and Biology

\section{MOLECULAR EPIDEMIOLOGY OF SEROTYPE 1 PNEUMOCOCCUS IN SOUTH AFRICA, 1991-2012}

M. du Plessis ${ }^{1}$, K.A. Jolley ${ }^{2}$, K. Ndlangisa ${ }^{1}$, N. Wolter ${ }^{1}$, S. Govindpershad ${ }^{1}$, L. de Gouveia ${ }^{1}$, D. Everett ${ }^{3}$, S.D. Bentley ${ }^{4}$, K.P. Klugman ${ }^{5}$, A. von Gottberg ${ }^{1}$

${ }^{1}$ Centre for Respiratory Diseases and Meningitis, National Institute for Communicable Diseases, Johannesburg, South Africa; ${ }^{2}$ Department of Zo ology, University of Oxford, Oxford, United Kingdom; ${ }^{3}$ Malawi-Liverpool-Wellcome Trust, Clinical Research Program, Blantyre, Malawi; ${ }^{4}$ Pathogen Genomics, Wellcome Trust Sanger Institute, Hinxton, United Kingdom; ${ }^{5}$ Hubert Dept. of Global Health Rollins School of Public Health and Division of Infectious Diseases School of Medicine, Emory University, Atlanta, USA

Background: PCV7 was introduced in 2009 and transitioned to PCV13 in 2011. Three geographically distinct lineages have been described for serotype 1: ST-306 (Europe and USA), ST-217 (Africa and Israel), ST-615 (Chile). We describe genotypes and temporal diversity among invasive serotype 1.

Methods: Isolates collected from children $(<5 y)$ and adults in 2007 and 2011 (pre-PCV13, n=328), and 2012 (early post-PCV13, n=155), were selected for MLST. An additional 142 isolates (1991-2011) were selected for genome sequencing. Sequence types (ST), genome data and ST diversity were analysed using eBURST, rMLST BIGSdb genome database (http://rmlst.org/) and Simpson's diversity index (D), respectively.

Results: MLST data were available for $94 \%(590 / 625)$ of isolates. In children ( $n=146)$, pre-PCV13, ST-217 ( $n=91)$ and two SLVs $(n=27)$ represented $96 \%(118 / 123)$ of isolates. Post-PCV13, ST-217 $(n=10)$ and three SLVs ( $n=12)$, including new ST-9067, represented 96\% (22/23). ST diversity, post-PCV13, was greater compared to pre-PCV13 [D=0.72 $(95 \% \mathrm{Cl}, 0.62-0.83)$ vs $\mathrm{D}=0.41(95 \% \mathrm{Cl}, 0.32-0.51)]$. In older individuals $(n=444)$, pre-PCV13, ST-217 $(n=242)$ and seven SLVs $(n=69)$ represented 95\% (311/326). Post-PCV13, ST-217 $(n=80)$ and three SLVs $(n=34)$, including ST-9067, represented $97 \%(114 / 118)$. ST diversity, pre- and post-PCV13, was similar with $\mathrm{D}=0.42(95 \% \mathrm{Cl}, 0.36-0.48)$ and 0.52 $(95 \% \mathrm{Cl}, 0.41-0.62)$, respectively, although ST composition differed. Genomic MLST analysis of pre-PCV13 ST-217 isolates confirmed clonality, stratified by age group or year, although 1991 isolates $(n=5)$ clustered separately. Conclusion: ST-217 and associated SLVs accounted for almost all serotype 1 disease. Serotype 1 diversity increased among children post-PCV13. Most notable was the emergence of new ST-9067 in all ages in 2012.

No conflict of interest 


\title{
CO-COLONIZATION AND ANTIBIOTIC SUSCEPTIBILITY OF STREPTOCOCCUS PNEUMONIAE AND GROUP B STREPTOCOCCI AMONGST GAMBIAN INFANTS PRIOR TO PCV13 VACCINATION
}

E. Foster-Nyarko ${ }^{1}$, B. Kwambana ${ }^{1}$, A. Bojang ${ }^{1}$, F. Ceesay ${ }^{1}$, O. Ogundare ${ }^{1}$, O. Aderonke ${ }^{1}$, I. Adetifa ${ }^{2}$, M. Ota ${ }^{3}$, M. Antonio $^{1}$

${ }^{1}$ Vaccinology, Medical Research Council Unit The Gambia, Banjul, Gambia; ${ }^{2}$ Disease Control \& Elimination, Medical Research Council Unit The Gambia, Banjul, Gambia; ${ }^{3}$ Regional Office for Africa, World Health Organization, Brazzaville, Republic of Congo

Background and Aims: Nasopharyngeal co-colonization of Streptococcus pneumoniae and Group B Streptococci (GBS) is unknown in Gambian infants. We investigated the co-carriage of S. pneumoniae, GBS, Staphylococcus aureus and Moraxella catarrhalis amongst Gambian infants prior to PCV13 vaccination.

Methods: 1200 nasopharyngeal swabs were collected from infants aged 2-3 months prior to administration of pneumococcal and Haemophilus influenzae type B (Hib) conjugate vaccines. Microbiological methods were used to culture S. pneumoniae, GBS, Hi, S. aureus and M. catarrhalis. Antimicrobial susceptibility testing was determined by Kirby-Bauer method.

Results: The prevalence of S. pneumoniae and GBS were $62 \%(95 \% \mathrm{Cl}: 59 \%-65 \%)$ and $14 \%(95 \% \mathrm{Cl}: 12 \%-16 \%)$ respectively. Of the 744 infants that had S. pneumoniae, $72(10 \%)$ had co-carriage with GBS. The carriage of $S$. pneumoniae was negatively associated with GBS (OR $0.43[95 \% \mathrm{Cl}: 0.31-0.60], \mathrm{p}<0.001$ ); and positively associated with $M$. catarrhalis (OR 1.52 [95\% $\mathrm{Cl}: 1.18-1.96], p=0.001$ ) and $\geq 3$ other children $<5$ years old sleeping in the same room (OR 1.86[95\%Cl: 1.20-2.81], $\mathrm{p}=0.005$ ). However, the carriage of GBS was positively associated with $S$. aureus (OR 1.89[95\% Cl: 1.33-2.69], $\mathrm{p}<0.001$ ) and negatively associated with $\mathrm{M}$. catarrhalis (OR 0.61 [95\% $\mathrm{Cl}: 0.40-0.92]$, $\mathrm{p}=0.017$ ).

Susceptibility of S. pneumoniae to penicillin, chloramphenicol, tetracycline and erythromycin were $82 \%, 96 \%, 59 \%$ and $92 \%$ respectively. Those of GBS were $100 \%, 99 \%, 91 \%$ and $85 \%$ respectively.

Conclusion: Our preliminary data shows a negative association between S. pneumoniae and GBS in Gambian infants. This may have important clinical implications for species replacement post pneumococcal vaccination.

No conflict of interest

ISPPD-0371

The Promiscuous Pneumococcus---Evolution and Biology

\section{SEROTYPE DISTRIBUTION AND ANTIMICROBIAL RESISTANCE OF INVASIVE STREPTOCOCCUS PNEUMONIAE ISOLATES IN SERBIA, 2012-2013}

\author{
I. Gajic ${ }^{1}$, V. Mijac ${ }^{1}$, L. Ranin'1 , Z. Vasiljevic ${ }^{2}$, G. Lazarevic ${ }^{3}$, N. Opavski ${ }^{1}$
}

${ }^{1}$ Bacteriology Department, Institute of Microbiology and Immunology, Belgrade, Serbia; ${ }^{2}$ Microbiology Department, Institute for mother and child health care of Serbia "Dr Vukan Cupic", Belgrade, Serbia; ${ }^{3}$ Microbiology Department, University Children's Hospital in Belgrade, Belgrade, Serbia

Background and Aims: Pneumococcal conjugated vaccines (PCV) have been registered, but not reimbursed in Serbia. The aim of this study was to determine serotypes and PCV coverage of invasive Streptococcus pneumoniae in Serbia.

Methods: A total of 78 invasive S. pneumoniae isolates ( 25 from children; 53 from adults) were collected throughout country during the period January 2012-October 2013. The serotypes, antimicrobial susceptibility and macrolide resistance genes were determined using Quellung reaction, disk diffusion test and PCR, respectively. Results: Ten serotypes accounted for $79.5 \%$ of the isolates $(3,4,14,7 \mathrm{~F}, 19 \mathrm{~F}, 23 \mathrm{~F}, 22 \mathrm{~F}, 9 \mathrm{~V}, 9 \mathrm{~N}$ and 8$)$, among which the most prevailing was serotype $3(20.5 \%)$. The most common serotypes among children $\leq 5$ years were: $19 \mathrm{~F}$ $(15.8 \%)$ and $23 \mathrm{~F}(15.8 \%)$, while serotype 3 predominated among adults $\geq 65$ years $(27.8 \%)$. The serotype coverage of 7-valent, 10-valent, and 13-valent PCV for isolates originated from children <5years was: $52.6 \%, 68.4 \%$ and $84.2 \%$, respectively. Penicillin non-susceptibility was strongly associated with serotypes 14, 23F and 19F. Penicillin and cefotaxime/ceftriaxone resistance for meningitis strains were $44 \%$ and $18.5 \%$, while for non-meningitis isolates were $11 \%$ and $4.9 \%$, respectively. Among 17 macrolide resistant isolates $(21.8 \%), 64.7 \%$ harbored ermB gene and $35.3 \%$ mefA. Co-resistance to erythromycin and penicillin was $9 \%$ of strains. All isolates were fully susceptible to vancomycin, linezolid, fluoroquinolones, telithromycin and rifampicin.

Conclusion: The most common serotypes causing invasive pneumococcal diseases in Serbia were: 3, 4, 14, 7F, $19 \mathrm{~F}$ and $23 \mathrm{~F}$. PCV-7, PCV-10 and PCV-13 vaccine coverage in children $\leq 5$ years were $52.6 \%, 68.4 \%$ and $78.9 \%$, respectively.

No conflict of interest 


\title{
GENETIC DIVERSITY OF STREPTOCOCCUS PNEUMONIAE SEROGROUP 6 IN KILIFI, KENYA
}

\author{
S.P. Henson ${ }^{1}$, C.E. Moore ${ }^{2}$, S.C. Morpeth ${ }^{1}$, A. Karani ${ }^{3}$, S. Nyongesa ${ }^{1}$, D.W. Crook ${ }^{2}$, J.A.G. Scott ${ }^{1}$ \\ ${ }^{1}$ Epidemiology and Demography, KEMRI-Wellcome Trust Research Programme, Kilifi, Kenya; ${ }^{2}$ Nuffield Department of Clinical Medicine, Univer- \\ sity of Oxford, Oxford, United Kingdom; ${ }^{3}$ Microbiology, KEMRI-Wellcome Trust Research Programme, Kilifi, Kenya
}

Background: Pneumococcal serotypes and sequence types (ST) are closely associated. The introduction of the pneumococcal conjugate vaccine (PCV) should select against STs that are associated with vaccine serotypes. Serotype 6B is included in PCV10 that was introduced in Kenya in 2011. We examined the change in proportions of serogroup 6 serotypes before and after vaccine introduction, and investigated the diversity among serogroup 6 isolates from Kilifi.

Methods: Invasive and carriage serogroup 6 isolates from hospital (Kilifi District Hospital) and community samples from children and adults collected between 1994 and 2013 were investigated. We serotyped, by Quellung and PCR 345 isolates and derived whole genome sequences, using the Illumina technology, for 57 isolates collected before 2008.

Results: The proportion of serotype $6 \mathrm{~B}$ among carriage isolates declined after vaccine introduction (9.7\% to $3.3 \%$; $\mathrm{p}<0.0001)$. The major STs circulating in Kenya differed from the major STs of serotype 6 strains circulating elsewhere in the world and there was considerable diversity in these STs. At the capsular locus, serotype 6B grouped into three clusters, with high between-cluster variation. Serotype 6A formed seven clusters but with less between-cluster variation. The most prominent serotype $6 \mathrm{~B}$ subtype, comprising $75 \%$ of isolates, is $6 \mathrm{~B}-\mathrm{III}$, which is very similar to serotype 6E.

Conclusion: Although there is correlation between STs and serotype, we found isolates of unrelated STs had the same subtype. Streptococcus pneumoniae in Kilifi appears to be genetically diverse and distinct from wellcharacterized populations elsewhere. It is unclear whether this will affect the success of vaccination.

No conflict of interest

ISPPD-0114

The Promiscuous Pneumococcus---Evolution and Biology

\section{NanB AND NanC IN THE PATHOGENESIS OF SYSTEMIC PNEUMOCOCCAL INFECTION}

R. Janapatla ${ }^{1}$, M.H. Hsu' ${ }^{1}$, W.T. Liao ${ }^{1}$, C.H. Chiu ${ }^{2}$

${ }^{1}$ Chang Gung Memorial Hospital, Molecular Infectious Disease Research Center, Kweishan, Taiwan; ${ }^{2}$ Chang Gung Memorial Hospital Division of Pediatric Infectious Diseases Department of Pediatrics Chang Gung Children's Hospital Chang Gung University College of Medicine, Molecular Infectious Disease Research Center, Kweishan, Taiwan

Background and Aim: Pneumococcal neuraminidase NanA cleaves both $\alpha 2-3$ and $\alpha 2-6$ sialyl linkages, while both NanB and NanC can cleave only a2-3 sialyl linkages. In the present study we aimed to examine the role of the neuraminidases NanB and NanC in the pathogenesis of systemic pneumococcal infection.

Methods: Mutants of both nanB and nanC were constructed in Streptococcus pneumoniae serotype 6B by doublecrossover recombination mutagenesis. BALB/c mice were injected with $1 \times 10^{8}$ CFU S. pneumoniae in PBS through tail vein intravenously. Thomsen-Friedenrich antigen (T-antigen) exposure on the mice RBC was detected by flow cytometer.

Results: To monitor the influence of NanB and NanC on the survival of S. pneumoniae in mice bloodstream, BALB/c mice were intravenously challenged with wild type $S$. pneumoniae, nanB and nanC mutants. After specific time points day 1-, 2-, 3-, 5- and 7-, the blood was collected by retro- orbital puncture and subjected to bacterial culture. After 2 days infection, nanB mutant had a significantly lower bacteria counts in bloodstream than wild type $(p=$ 0.0207). nan B mutant had a significantly lower survival rate in bloodstream than wild type $(p=0.0340)$. nan $B$ and nanC mutants had a lower T-antigen exposure on the RBC than wild type. After wild type S. pneumoniae infection, prominent T-antigen exposure on the glomeruli was observed. After infection with nan $\mathrm{B}$ and nanC mutants, less T-antigen exposure on the glomeruli was observed.

Conclusion: In addition to NanA, NanB and NanC also play a role in pneumococcal infection. Although NanB and NanC can selectively release $\alpha 2-3$ sialyl linkages only, they can provide an additive effect along with NanA to expose Thomsen-Friedenrich antigen on various cells in host.

No conflict of interest 
ISPPD-0179

The Promiscuous Pneumococcus---Evolution and Biology

\title{
EPIDEMIOLOGY OF PNEUMOCOCCAL INFECTIONS AT THE FAR EAST OF RUSSIA
}

\author{
A. Martynova ${ }^{1}$, L.A. Balabanova², O. Chulakova ${ }^{3}$ \\ ${ }^{1}$ Far Eastern Federal University, State Pacific Medical University, Vladivostok, Russia; ${ }^{2}$ Marine Biochemistry, Pacific Institue of Bioorganic Chemis- \\ try, Vladivostok, Russia; ${ }^{3}$ State Pacific Medical University, Epidemiology department, Vladivostok, Russia
}

Background: Pneumococcal infections remain major public health problem worldwide for all age groups, and also for aged population. There is still no molecular epidemiological characteristic of Streptococcus pneumoniae isolates gained from aged people with different forms of pneumococcal infections.Aims: was to perform multilocus sequence typing (MLST) in strains of S. pneumoniae gained in patients elder of 60 years at the territory of Primorsky region as the most southern part of the Russian Far East.

Methods: MLST was conducted with housekeeping genes on standard method on recommendations of MC Enright (1998) et al. with previous study of antimicrobial agents resistance and serotyping.

Results: There were taken 50 isolates ( group 1, from patients with community-acquired pneumonia), 30 isolates ( group 2, from patients with invasive pneumococcal infections such as bacteremia and meningitis). Isolates form 1 st group included 24 serotypes ( 26 of non-typable) and 22 sequence types ( ST) according to MLST of which 7 were novel, 8 were of Taiwanese clones (TW-28,19,26) of 19F serotype, 3 were of R6, EU38, SP95. In group 2 of 20 strains there was revealed 8 serotypes ( 12 of non-typable) and 6 ST of Taiwanese clones. In strains from patients there were isolated $18 \%$ resistant to erythromycin, $16 \%$ tetracycline, $4 \%$ chloramphenicol. The pattern of antimicrobial agents resistance from the strains of 2 nd group was $35 \%$ to erythromycin, $15 \%$ to tetracycline, $5 \%$ to fluoroquinolones.

Conclusion: Multilocus sequence typing allows us to suggest the epidemiological significance of Taiwanese isolates of $S$. pneumoniae in our region.

No conflict of interest

ISPPD-0150

The Promiscuous Pneumococcus---Evolution and Biology

\section{SEROTYPE DIVERSITY OF THE PMEN CLONES BEFORE AND AFTER THE INTRODUCTION OF 13-VALENT PNEUMOCOCCAL CONJUGATE VACCINE (PCV13) IN CATALONIA, SPAIN}

\author{
N. Peris ${ }^{1}$, L. Selva ${ }^{1}$, C. Muñoz-Almagro ${ }^{1}$, On Behalf of Catalan Study Group of Invasive Pneumococcal Disease \\ ${ }^{1}$ Molecular Microbiology, Hospital Sant Joan de Deu, Esplugues Barcelona, Spain; ${ }^{2}$ Cooperative study group, ., Catalonia, Spain
}

Background: PMEN (Pneumococcal Molecular Epidemiology Network) clones include 26 multidrug-resistant and 17 susceptible Streptococcus pneumoniae clones that are a recognized cause of pneumococcal disease and have widespread geographical impact.

Aims: The aim of the study is to analyze the serotypes diversity of PMEN clones among pneumococcal invasive isolates before and after the introduction in 2010 of PCV13 vaccine in Catalonia.

Methods: Prospective study which included all pneumococcal invasive isolates received in the Catalonia Support Molecular Laboratory for Invasive Pneumococci. All isolates were characterized by serotyping and clonal type. Genetic diversity of isolates was estimated using the Simpson's index of diversity. Periods 2009-2010 and 2011-2012 were considered prevaccine and vaccine period respectively.

Results: A total of 1831 invasive pneumococci were registered and 1810 (98.8\%) were viable for serotype and clonal study. 57.2\% were isolated during 2009-2011 and 42.8\% during 2011-2012. 20.3\% were isolated in patients $<5$ years old. PCV13 serotypes and PMEN clones were detected in 1131 and 759 isolates respectively. PCV13 serotypes decreased from $69.3 \%$ during prevaccine-period to $53.3 \%$ during vaccine-period $(p<0.001)$. PMEN clones declined from $44.1 \%$ to $39.1 \%(p=0.03)$. Among PMEN clones non-PCV13 serotypes increased from $10.1 \%$ during prevaccine-period to $19.5 \%$ during vaccine-period $(p<0.001)$. A rise of serotype diversity among PMEN clones was observed comparing the two periods: Simpson's index of diversity of 0.821 (95\% Cl 0.799-0.843) vs. 0.857 (95\% Cl 0.837-0.877).

Conclusion: An increase of serotype diversity among PMEN clones due to an increase of non-PCV13 serotypes has been observed after the introduction of PCV13 vaccine in Catalonia.

No conflict of interest 
ISPPD-0392

The Promiscuous Pneumococcus---Evolution and Biology

\title{
MOLECULAR INVESTIGATIONS ON DISTRIBUTION OF VARIOUS VIRULENCE FACTORS AMONG THE ISOLATES OF STREPTOCOCCUS PNEUMONIAE OBTAINED FROM DIFFERENT DISEASE ETIOLOGIES
}

J. John ${ }^{1}$, K. Kasudhan ${ }^{1}$, S. Sah ${ }^{1}$, P. K ${ }^{1}$

${ }^{1}$ Department of Biotechnology, Pondicherry University, Pondicherry, India

Background and Aims: Streptococcus pneumoniae that causes pneumonia and other invasive diseases in elderly and children continues to be a major cause of morbidity and mortality in developing countries including India and there is paucity of data in India on pathogenesis of S. pneumoniae. We attempted the characterization of S. pneumoniae isolates through analyzing the distribution of virulence markers among the isolates cultured from different disease etiologies.

Methods: A total of 37 isolates obtained from different Southern Indian hospitals were investigated by polymerase chain reaction (PCR) for detecting different virulence genes such as lytA coding for autolysin, an enzyme that degrades host cell-wall, $p l y$ that codes for pneumolysin toxin, $p s p A$ encoding pneumococcal surface protein-A involved in immune evasion and eno coding for anchorless surface protein enolase having proteolytic activity. Genes like $p s a \mathrm{~A}$, amiA coding for adhesion proteins, a regulatory gene ciaR and finally, an essential protein that brings in capsule variation coded by wchA was also checked.

Results: Majority of isolates were PCR positive for ciaH and amiA (77\%; n=27) where as almost all had autolysin lytA gene except one isolate (BJMC5). Sixty three percent isolates were positive for ply gene $(n=22)$ and surface protein genes $p s a \mathrm{~A}$, eno were detected in 23 isolates (66\%). pbpA was seen in 22 isolates $(63 \%)$ and wchA presence was observed only in $10(29 \%)$ isolates.

Conclusion: Presence of autolysin, regulatory and adhesion proteins such as $\mathrm{CiaH}$ and AmiA appear to be very critical for pathogenesis of $S$. pneumoniae. This study has helped in understanding the extent of contribution of different virulence determinants for the S. pneumoniae pathogenesis.

No conflict of interest

ISPPD-0470

The Promiscuous Pneumococcus---Evolution and Biology

\section{SCREENING OF STREPTOCOCCUS PNEUMONIAE ISOLATES FOR WChA POINT MUTATIONS THAT ARE RESPONSIBLE FOR IMPAIRED CAPSULAR SYNTHESIS}

\author{
P. K ${ }^{1}$, J. John ${ }^{1}$, K. Kasudhan ${ }^{1}$, S. Sah ${ }^{1}$ \\ ${ }^{1}$ Department of Biotechnology, Pondicherry University, Pondicherry, India
}

Background and Aims: Streptococcus pneumoniae is one of the important pathogen causing pneumonia and other invasive diseases in elderly and children. The present study attempted a screening of $S$. pneumoniae isolates for the presence of wchA gene as well investigated the occurrence of point mutations in wchA that has been implicated for the non-typability of $S$. pneumoniae isolates typically isolated from nasopharyngeal carriage or from conjunctivitis. Methods: A total of 37 isolates obtained from different South Indian hospitals were investigated by polymerase chain reaction (PCR) for the presence of wchA gene that encodes for membrane-associated protein WchA, which has the activity of glucosyl-1-phosphate transferase that participate in capsular polysaccharide synthesis. Point mutations in the wchA have been shown to bring in capsule variation in pneumococci. All amplified wchA amplicons from different isolates were custom sequenced and analyzed for point mutations after performing multiple alignments of DNA sequences of wchA genes available in Genbank

Results: Twelve isolates showed amplification of wchA gene among all the isolates tested. Neighbor joining tree deduced from multiple alignments of wchA through comparing the DNA sequences from all the 12 isolates showed four clusters. Analysis of isolates belonged to cluster-3 comprising of three isolates namely CMRI-1, CMRI-7 and BMP 241-13 revealed significant results. Three important point mutations appeared to be common in all the three isolates, when compared with the referral strain 554/62 wchA sequence (GenBank accession no. CR931643) at three different positions such as 31, 239 and 390. In position 31, there was a change in amino acid from A $\rightarrow>T$, at position 239, there was a amino acid change $\mathrm{Y}->\mathrm{H}$ and finally, at 319 position the amino acid change was $\mathrm{S}->\mathrm{I}$. Conclusion: Three of the $S$. pneumoniae isolates having mutations at the identical position appeared to be very significant and interestingly all these isolates were cultured from ocular infections. The present study reveals the possible variation in capsular nature that is likely to be contributing to the $S$. pneumoniae pathogenesis in ocular infections. The significance of these mutations has to be further analyzed to get better insights on these events of point mutations.

No conflict of interest 


\title{
INTER- AND INTRA- PMEN CLONES DIFFERENCES IN THE GROWTH IN SUPPLEMENTED TODD HEWITT BROTH
}

\author{
B. Quintero ${ }^{1}$, E. Alfonzo ${ }^{2}$, M. Araque ${ }^{2}$, C. van der Gaast- de Jongh ${ }^{3}$, P.W.M. Hermans ${ }^{3}$ \\ ${ }^{1}$ Department of Microbiology and Parasitology. Faculty of Medicine, Los Andes University, Merida, Venezuela; ${ }^{2}$ Department of Microbiol- \\ ogy and Parasitology. Faculty of Pharmacy and Bioanalysis, Los Andes University, Merida, Venezuela; ${ }^{3}$ Laboratory of Pediatric Infectious Diseases, \\ Radboud University Nijmegen Medical Centre, Nijmegen, Netherlands
}

Background and Aim: Pneumococcal serotypes and clones differ in colonization prevalence and the likelihood of causing disease. It has been suggested that invasiveness and colonization prevalence of Streptococcus pneumoniae could be associated with in vitro growth. We compared in vitro growth among pneumococcal strains with serotypes and PMEN clones with different colonization prevalence and invasive potential.

Methods: In vitro growth was measure in 6 isolates colonizing asymptomatic children in Mérida, Venezuela related with NCC1 acapsular clon, and PMEN England ${ }^{14}-9$ and Tennessee ${ }^{23 F}-4$ clones. Strains were grown in Todd Hewitt Broth (THB) with or without $0.4 \%$ glucose and $5 \%$ sheep blood (SB), and optical densities were measured every hour for 16 hours.

Results: Growth in THB showed inter and intra-clonal statistical differences $(p=0.001)$. Supplementations with glucose, SB and glucose+SB stimulated an acceleration of cellular growth after 7, 3 and 2 hours of incubation, respectively. THB supplemented with of glucose, SB and glucose+SB increased cellular growth significantly $(p=$ 0.001 ) in 1.5, 5 and 7 times, respectively, compared with non-supplemented THB.

Conclusion: These results suggest that variations in growth between the pneumococcal strains might respond to different characteristics other than serotype or MLST type. It is well known that the delay in the diagnosis of pneumococcal disease increases sequels and mortality. This research also shows that THB supplemented with glucose and SB reduces the time for the recovery of pneumococcal strains. Usefully, this would allow the microbiology laboratory make faster reports and, consequently, improve the clinical outcome of patients with pneumococcal disease.

No conflict of interest

ISPPD-0256

The Promiscuous Pneumococcus---Evolution and Biology

\section{VIRAL CO-INFECTIONS AMONG ADULTS HOSPITALIZED WITH PNEUMOCOCCAL-ASSOCIATED ACUTE LOWER RESPIRATORY INFECTIONS (ALRI) IN RURAL THAILAND, 2004-2012}

J. Rhodes ${ }^{1}$, M. Chittaganpitch ${ }^{2}$, S. Thamthitiwat ${ }^{1}$, S. Henchaichon ${ }^{1}$, B. Piralam ${ }^{3}$, P. Sawatwong ${ }^{1}$, S. Chantra ${ }^{4}$, C. Grego$\mathrm{ry}^{1}$, S. Olsen ${ }^{5}, \mathrm{H}$. Baggett ${ }^{1}$

${ }^{1}$ International Emerging Infections Program, Thailand MOPH - US CDC Collaboration, Nonthaburi, Thailand; ${ }^{2} \mathrm{National}$ Institute of Health, Thailand Ministry of Public Health, Nonthaburi, Thailand; ' ${ }^{3}$ EIP Nakhon Phanom, Provincial Health Office, Nakhon Phanom, Thailand;

${ }^{4}$ Sa Kaeo Crown Prince Hospital, Provincial Health Office, Sa Kaeo, Thailand; ${ }^{5}$ Influenza Division, US Centers for Disease Control and Prevention, Atlanta, USA

Background: We examined viral-pneumococcal co-infections in rural Thailand where vaccination coverage for influenza is low, PCV is not included in routine vaccinations, and the June-October pneumonia season differs from typical winter peaks observed in temperate climates.

Methods: Surveillance for acute lower respiratory infections (ALRI) hospitalizations was conducted in all hospitals of two Thai provinces (population 1.2 million). Systematically selected subsets of ALRI patients aged $>17$ years had nasopharyngeal specimens tested for viruses by PCR and urine tested for pneumococcal antigen. Blood cultures were performed as clinically indicated.

Results: Among 13,866 patients hospitalized with ALRI, $4.3 \%(n=593)$ had confirmed pneumococcal infections, of which 555 (94\%) were detected by urine antigen only. Pneumococcal positivity ranged from $4 \%$ to $9 \%$ among ALRI cases with $>1$ virus detected: adenovirus $3.9 \%$, influenza A/B viruses $4.0 \%$, respiratory syncytial virus (RSV) $4.5 \%$, human metapneumovirus (HMPV) 7.8\%, and parainfluenza viruses (HPIV) 8.6\%. Among the 593 pneumococcal cases, $93(16 \%)$ had $>1$ virus detected. Nearly half (46\%) of pneumococcal cases without viral co-infections occurred during a single annual peak in Dec-Mar, when adenovirus and HPIV peaked; however, viral-pneumococcal coinfections were concentrated around Aug-Oct (50\%), when influenza, RSV, and HMPV peaked. Disease severity was similar between pneumococcal cases with and without viral co-infections: $61 \%$ vs. $56 \%$ required supplemental oxygen; $8.7 \%$ vs. $9.0 \%$ required intubation; and $5.4 \%$ vs. $5.6 \%$ died.

Conclusion: Viral co-infections are common among adults with pneumococcal ALRI, suggesting that preventing viral infections could complement primary pneumococcal prevention strategies. Non-coincident pneumococcal and influenza seasons offer a unique opportunity to examine individual and co-infections.

No conflict of interest 


\title{
IN VIVO TRANSCRIPTOMIC ANALYSIS USING RNA-SEQ OF PNEUMOCOCCAL GENES EXPRESSED IN THE PLEURAL CAVITY
}

\author{
N.D. Ritchie ${ }^{1}$, S.C. Irvine ${ }^{1}$, T.J. Evans ${ }^{1}$ \\ ${ }^{1}$ Infection Immunity \& Inflammation, University of Glasgow, Glasgow, United Kingdom
}

Introduction: Specific patterns of gene expression allow the pneumococcus to occupy different niches within the host. We set out to examine what genes were differentially expressed in the pleural cavity following in a murine model of pneumonia

Methods: Mice were infected by the intranasal route with strain SRL1 (serotype1) that resulted in pneumonia and pleural infection. Bacteria recovered from the pleural space were treated immediately with Trizol and RNA purified. RNA was similarly purified from SRL1 grown in broth. Following library construction, Paired-end RNA transcripts were sequenced using a Hi-Seq and analysed using CLC Genomics Workbench. Changes in transcript levels between pleura and broth were assessed for statistical significance using Kal's z test; fold-changes $>2$ with Bonferroni corrected $p<0.05$ were classified as significant.

Results: Sequence reads in excess of 15 million were obtained from both samples. 983 genes were significantly up-regulated ( $2-40$ fold) in pleura compared to broth and 278 were down-regulated ( $2-32$ fold). Upregulated genes included the blp/pnc operon encoding bacteriocin and related genes, purine biosynthetic genes, the $\mathrm{N}$-acetyl glucosamine specific phosphotransferase system and those controlling competence. Down-regulated genes included many ribosomal proteins, the htrA protease, genes controlling galactose metabolism and the $\mathrm{H}_{2} \mathrm{O}_{2}$ producing enzyme spxB.

Conclusion: RNA-seq is a powerful tool to identify in vivo patterns of gene expression. Many genes identified as upregulated in the pleura were not previously known to be important in virulence. Further work will define what roles they play in establishing pleural infection.

No conflict of interest

ISPPD-0417

The Promiscuous Pneumococcus---Evolution and Biology

\section{INFLUENZA A VIRUS-MEDIATED PRIMING OF LUNG INNATE IMMUNITY ENHANCES CYTOKINE SECRETION OF LUNG CELLS AND INHIBITS CLEARANCE OF STREPTOCOCCUS PNEUMONIAE}

\author{
V. Sender ${ }^{1}$, B. Henriques-Normark ${ }^{1}$
}

${ }^{1}$ Microbiology Tumor and Cell Biology, Karolinska Institute, Stockholm, Sweden

Both, influenza A virus and Streptococcus pneumoniae are a leading cause of morbidity and mortality worldwide. However, most influenza-related mortality is not due to the viral infection alone but rather secondary bacterial infections, mainly caused by S. pneumoniae. The mechanisms driving virulent influenza co-infection are poorly defined, making it difficult to develop effective therapeutic strategies. This study investigates early signaling events evoked by influenza infections affecting the innate immune response and cellular clearance mechanisms in the lung. Using an in vivo model of subsequent infections with influenza A virus and S. pneumonia via the intratracheal infection route we show that sublethal influenza infections clearly predispose for severe pneumococcal infections even at low bacterial doses. Co-infected C57BL/6 mice are more susceptible to pneumococcal infection than single-infected mice, resulting in drastically less survival and earlier development of pneumonia and septicemia. Preliminary flow cytometry data on kinetic changes of cell populations in lavage fluid and post-lavage lung tissue from single and co-infected animals, as well as viable bacterial and viral counts in lungs suggest that virus-induced innate defects impair early bacterial clearance, thereby supporting the development of secondary bacterial pneumonia. Cytokine analyses reveal an up-regulation of IL- 6 and TNF- $\alpha$ in bronchoalveolar lavage from co-infected mice compared to single-infected mice. Further investigations of lung cell populations, their possible cross-activation, and reduced clearance potential during influenza-pneumococcal co-infections will increase our understanding of the mechanisms behind influenza-mediated sensitization of the host to pneumococcal infections which will help to find treatment regimens that combat secondary bacterial infections.

No conflict of interest 


\title{
NEXT-GENERATION SEQUENCING ANALYSIS OF A PUTATIVE IRON-RESPONSIVE TRANSCRIPTIONAL REGULATOR MUTANT IN STREPTOCOCCUS PNEUMONIAE TIGR4
}

\author{
T. Tower ${ }^{1}$, R. Gupta ${ }^{2}$, E. Swiatlo², B. Nanduri ${ }^{1}$ \\ ${ }^{1}$ College of Veterinary Medicine, Mississippi State University, Starkville, USA; ${ }^{2}$ Department of Microbiology, University of Mississippi Medi- \\ cal Center, Jackson, USA
}

Iron is an essential micronutrient for many pathogenic bacteria and plays an important role in gene regulation. Cellular response to environmental iron is critical for pathogens such as Streptococcus pneumoniae that inhabit multiple anatomical sites in the host, since both form and quantity of available iron differ greatly in each location. The genome of serotype 4 pneumococcal strain TIGR4 encodes a putative iron-dependent transcriptional regulator (Idtr). In this study we used the next-generation sequencing (NGS) Illumina MiSeq platform to analyze differential gene expression between TIGR4 and an isogenic mutant deleted at idtr ( $\Delta i d t r$ ) cultured in vitro in both high and lowiron conditions. Total RNA was collected and rRNA was removed before reverse transcription and sequencing. The RNA-Rocket software suite was used to align the reads and identify significant changes in gene expression. A total of 291 genes were identified as differentially-expressed at \pm 1.5 -fold difference. These genes included choline-binding proteins, heat shock and stress- induced proteins, as well as multiple sugar transport and metabolism genes. These data suggest that intracellular iron levels may affect the regulation of pneumococcal gene expression in different environmental niches in which iron availability varies widely.

No conflict of interest

ISPPD-0144

The Promiscuous Pneumococcus---Evolution and Biology

\section{MOLECULAR BIOLOGICAL CHARACTERIZATION OF NON-TYPEABLE (NT) STREPTOCOCCUS PNEUMONIAE ISOLATES}

M. van der Linden ${ }^{1}$, D. Ajah ${ }^{1}$, S. Schlösser ${ }^{1}$, T. Vierbuchen ${ }^{1}$, S. Zwirner ${ }^{1}$, J. Otten ${ }^{1}$, N. Levina ${ }^{1}$, C. Heeg ${ }^{1}$, M. Jaschek $^{1}$, M. Imöhl ${ }^{1}$

${ }^{1}$ National Reference Center for Streptococci Department of Medical Microbiology, University Hospital Aachen, Aachen, Germany

Background and Aims: The German National Reference Center for Streptococci (GNRCS) has been carrying out surveillance of invasive pneumococcal disease since 1992. Of a total of 37,000 pneumococcal isolates, about 400 were classified as non-typeable (NT). NT isolates are not covered by any of the current vaccines and may have the intrinsic capacity of being replacement serotypes. Additionally, they form the pool in which 'new serotypes' might be detected. Therefore, in this study we (re-)analyzed these isolates using modern molecular techniques.

Methods: Identification of the isolates was re-assessed using our current expertise with the Quellung method, PCR of the lytA-gene followed by restriction-analysis, and sequencing of the sodA-gene. MLST, multiplex-PCR, B $\mu \mathrm{GGS}$ array and genomic-sequencing were used for identification and characterization of the NT isolates.

Results: A total of 404 NT isolates were analysed. Of these, 114 (28.2\%) were not Streptococcus pneumoniae - in part identified as S. pseudopneumoniae, 137 isolates (33.9\%) were typeable using the Quellung method, most of them with rare serotypes $(27,29,31,34,37,38) .123$ isolates $(30.4 \%)$ were really non-typeable, while 16 isolates were not viable (14 isolates have not been assessed so far). MLST of 54 real non-typeable isolates showed that 35 had STs occurring in mlst.net with reported serotypes. Microarray-analyses, genomic-sequencing and electronmicroscopy revealed point-mutations resulting in defective capsular-cassettes.

Conclusion: Reassessment of NT-isolates might be useful as they may represent rare serotypes that were previously undetected. Among the remaining real non-typeable isolates some were identified having point-mutations in their capsular-genes. These isolates would have been falsely assigned serotypes using PCR-methods.

No conflict of interest 
ISPPD-0418

The Promiscuous Pneumococcus---Evolution and Biology

\title{
EPIDEMIOLOGICAL CHARACTERIZATION OF STREPTOCOCCUS PNEUMONIAE USING MULTI LOCUS SEQUENCE TYPING (MLST) ACROSS INDIA
}

\author{
R. Varghese ${ }^{1}$, R. Jeyaraman ${ }^{1}$, B. Veeraraghavan ${ }^{1}$, K. Thomas ${ }^{2}$ \\ ${ }^{1}$ Microbiology, Christian Medical College, Vellore, India; ${ }^{2}$ General Medicine, Christian Medical College, Vellore, India
}

Background and Aim: Streptococcus pneumoniae is a major cause of invasive bacterial disease in both children and adults worldwide especially in India. Geographical diversity of serotypes and serotype exchange (capsular switching) in S.pneumoniae limits the efficacy of new conjugate vaccines produced against limited number of serotypes. So serotyping and molecular typing has to go hand in hand to characterize pneumococcal population. In molecular typing MLST provides unambiguous data that are portable and comparable between the laboratories which in turn help us understand the population and evolution biology of these organisms. In these circumstances we commenced this study with the aim to identify the common invasive sequence types (using MLST) present over different regions of India and to check for correlation between serotypes and sequence types.

Materials and Methods: 40 invasive S.pneumoniae isolates from children $<5$ years, collected from different sites within India was serotyped by Co-agglutination and reconfirmed by PCR, followed by MLST. Antimicrobial susceptibility profile was done using Vitek II system.

Results: The most frequent serotypes were 6B, 14, 4, 1,19F and 6A. The isolates showed $100 \%$ resistance to Cotrimoxazole followed by erythromycin (32.5\%) and penicillin (15\%). Serotypes $19 \mathrm{~F}$ and $6 \mathrm{~B}$ are common among the Penicillin resistant serotypes. Sequence types by MLST will be discussed during the presentation.

Conclusion: Continued surveillance by serotyping and sequence typing combined with antimicrobial susceptibility profile is imperative owing the diverse epidemiology, emergence of multi drug resistance and vaccine escape recombinants of S.pneumoniae.

No conflict of interest

ISPPD-0079

The Promiscuous Pneumococcus---Evolution and Biology

\section{COINFECTION WITH INFLUENZA A VIRUS EXACERBATES PNEUMOCOCCAL ACUTE OTITIS MEDIA}

J.T. Wren ${ }^{1}$, W.E. Swords ${ }^{1}$

${ }^{1}$ Department of Microbiology and Immunology, Wake Forest University, Winston-Salem, USA

Background and Aims: Streptococcus pneumoniae (pneumococcus) is both a nearly ubiquitous nasopharyngeal colonizer of children but also the leading cause of acute otitis media (AOM), accounting for billions of dollars in healthcare expenditures each year. Multiple factors have been proposed to be involved in this transition from colonization to disease; two of which include influenza A virus (IAV) and pneumococcal phase variation. Indeed, IAV is frequently associated with pneumococcal AOM and nasopharyngeal bacterial phase variation may influence otopathogen ascension to the middle ear. Thus, our aim was to investigate the role of pneumococcal-influenza synergism and bacterial phase variation in the pathogenesis of AOM.

Methods: Mice were infected intranasally with IAV followed by S. pneumoniae and the magnitude of nasal colonization, middle ear infection, and histopathological inflammation were assessed. Coinfection studies were also performed using isogenic pneumococcal phase variants.

Results: Preceding IAV infection enhanced the magnitude of pneumococcal colonization by nearly 9-fold. Inflammation and neutrophilic infiltration was likewise increased in the nasopharynx following IAV infection. Further, IAV established a direct correlation between nasal bacterial load and middle ear ascension, such that the magnitude of infection was increased by nearly 40 -fold. The effect of the phase of pneumococci in the nasopharynx on the IAV modulation of bacterial AOM is currently being studied.

Conclusion: Based on these studies, we conclude that the preceding IAV infection is inflammatory in nature, disrupting stable pneumococcal colonization and resulting in an enhanced nasal bacterial burden that is then associated with increased bacterial ascension into the middle ear.

No conflict of interest 
ISPPD-0057

The Promiscuous Pneumococcus---Evolution and Biology

\section{STUDIES ON PNEUMOCOCCAL ESTERASES}

H. Kahya ${ }^{1}$, P.W. Andrew ${ }^{1}$, H. Yesilkaya ${ }^{1}$

${ }^{1}$ Infection Immunity \& Inflammation, University of Leicester, Leicester, United Kingdom

Background and Aims: The pneumococcus has four putative esterase genes (SPD_0534 (estA), SPD_0932, SPD_1239, and SPD_1506). These lipolytic enzymes have been reported to be important for bacterial physiology and virulence in other microorganisms. However, no detailed study has been done on pneumococcal esterases to assess their role in pneumococcal biology. Hence, this study aims to investigate esterases' role in pneumococcal physiology and virulence.

Methods: Mariner mutagenesis was used for mutant construction. Total esterase activity was assayed by using chromogenic substrates. Metal affinity column was used to purify his-tagged recombinant proteins.

Results: The highest level of esterase activity could be obtained with p-NPA, indicating that the pneumococcus has esterase specific for short acyl chains. All mutants displayed significantly less esterase activity than the parental strain, and the highest reduction in activity was observed in SPD_0534 mutant. The purified EstA showed optimal activity against $\mathrm{p}$-NPA compared to other $\mathrm{p}$-nitrophenyl esters. Michaelis-Menten kinetics showed that Vmax and $\mathrm{Km}$ of EstA were $339.2 \mathrm{mU} \pm 6.07$ and $1.45 \mathrm{mM} \pm 0.16$, respectively. In addition, EstA activity against Bovine Sub-maxillary Mucin (BSM), highly acetylated substrate, showed that acetate release increased in a time and concentration dependent manner. We also showed that pre-treatment of BSM by EstA increases sialic acid release by neuraminidase.

Conclusion: All four genes contribute to total pneumococcal esterase activity though EstA is the major pneumococcal esterase. Pneumococcal esterases are specific for short acyl chains. EstA potentiate neuraminidase activity in vitro. Further work is underway to determine in vivo function of these enzymes.

No conflict of interest

ISPPD-0056

The Promiscuous Pneumococcus---Evolution and Biology

\section{STUDIES ON THE REGULATION OF PNEUMOCOCCAL PYRUVATE FORMATE LYASE}

F.A. Younis ${ }^{1}$, S. Shafeeq ${ }^{2}$, O.P. Kuipers ${ }^{2}$, P.W. Andrew ${ }^{1}$, H. Yesilkaya ${ }^{1}$

${ }^{1}$ Infection Immunity \& Inflammation, University of Leicester, Leicester, United Kingdom; ${ }^{2}$ Department of Molecular Genetics, University of Groningen, Groningen, Netherlands

Background and Aims: Streptococcus pneumoniae depends on carbohydrates to produce its metabolic energy. Galactose is the main source of sugar in the respiratory tract, and can be found within the structure of mucin. Fermentative metabolism of galactose leads to generation of mixed acids. The enzyme pyruvate formate lyase (PFL, coded by $p f$ ) is responsible for mixed acid fermentation and was also shown to be important for pneumococcal virulence. However, it is not known how pfl is regulated. Therefore, this study aims to investigate the transcriptional regulation of $p f$.

Methods: Microarray analysis of a pfl mutant was done. Metal affinity column was used to purify his-tagged recombinant proteins. SOEing-PCR was used for mutant construction. Electrophoretic mobility shift assays (EMSA) were done by using Molecular Probes fluorescence-based EMSA Kit (Invitrogen, UK).

Results: Differential expression of seven transcriptional regulators was identified in a pfl mutant relative to the wild type. It was hypothesised that the proteins encoded by these genes are responsible for either direct or indirect regulation of $p f$. EMSA results showed that three of these regulatory proteins bound to putative promoter region of $p f l$. To demonstrate the involvement of these regulators further in $p f l$ regulation, we mutated the transcriptional regulators and grew the mutants in the presence of glucose or galactose with or without oxygen, and analysed the end products. The mutants had different growth patterns, and end-product composition than the wild type strain. Conclusion: The results show that some of the transcriptional regulators studied, are involved in the regulation of pfl.

No conflict of interest 